\title{
FUZZY MULTIVALUED VARIATIONAL INCLUSIONS IN BANACH SPACES
}

\author{
S. S. CHANG, D. O’REGAN, AND J. K. KIM
}

Received 21 February 2005; Revised 20 April 2005; Accepted 29 June 2005

The purpose of this paper is to introduce the concept of general fuzzy multivalued variational inclusions and to study the existence problem and the iterative approximation problem for certain fuzzy multivalued variational inclusions in Banach spaces. Using the resolvent operator technique and a new analytic technique, some existence theorems and iterative approximation techniques are presented for these fuzzy multivalued variational inclusions.

Copyright (c) 2006 S. S. Chang et al. This is an open access article distributed under the Creative Commons Attribution License, which permits unrestricted use, distribution, and reproduction in any medium, provided the original work is properly cited.

\section{Introduction}

In recent years, the fuzzy set theory introduced by Zadeh [48] has emerged as an interesting and fascinating branch of pure and applied sciences. The applications of fuzzy set theory can be found in many branches of regional, physical, mathematical, differential equations, and engineering sciences, see [1-51]. Recently there have been new advances in the theory of fuzzy differential equations and inclusions [1, 3, 6, 25-29, 42]. Equally important is variational inequality theory, which constitutes a significant and important extension of the variational principle. Variational inequality theory provides us with a simple and natural framework to study a wide class of unrelated linear and nonlinear problems arising in pure and applied sciences. Recently, variational inequality theory has been extended and generalized in different directions, using novel and innovative techniques (in particular using the notion of the resolvent operator [37, 39]). A useful and important generalization of variational inequality theory is variational inclusions, which have been studied by Noor [33-37, 39-41], Chang et al. [10, 11, 13, 15], Siddiqi et al. [46], Chidume et al. [17], Gu [22], Huang et al. [24] (see also the references therein). Motivated and inspired by recent research work in these two fields Chang [8], Chang and Zhu [16] first introduced the concepts of variational inequalities for fuzzy mappings. Since then several classes of variational inequalities for fuzzy mappings were considered by Chang

Hindawi Publishing Corporation Journal of Inequalities and Applications Volume 2006, Article ID 45164, Pages 1-15 DOI 10.1155/JIA/2006/45164 
and Huang [14], Noor [33, 35, 38], Ding [18, 19], Park and Jeong [43, 44], Agarwal etal. [2, 3], Zhu et al. [50], Nanda [31], and Chang [12].

The purpose of this paper is to introduce the concept of general fuzzy multivalued variational inclusions in Banach spaces and to study the existence problem and the iterative approximation problem for certain fuzzy multivalued variational inclusions. Using the resolvent operator technique and a new analytic technique some existence theorems and iterative approximation techniques are established for these fuzzy multivalued variational inclusions. The results presented in this paper are new, and they generalize, improve, and unify a number of recent results, that is, the resolvent operator approach allows us to obtain a more general theory (e.g., the results in [33-41, 43, 44, 47-49] are special cases of our main result).

\section{Preliminaries}

Throughout this paper, we assume that $E$ is a real Banach space with a norm $\|\cdot\|, E^{*}$ is the topological dual space of $E, \mathrm{CB}(E)$ is the family of all nonempty bounded and closed subsets of $E, D(\cdot, \cdot)$ is the Hausdorff metric on $\mathrm{CB}(E)$ defined by

$$
D(K, B)=\max \left\{\sup _{x \in K} d(x, B), \sup _{y \in B} d(K, y)\right\}, \quad K, B \in \mathrm{CB}(E)
$$

$\langle\cdot, \cdot\rangle$ is the dual pair between $E$ and $E^{*}, D(T)$ and $R(T)$ denote the domain and range of an operator $T$, respectively, and $J: E \rightarrow 2^{E^{*}}$ is the normalized duality mapping defined by

$$
J(x)=\left\{f \in E^{*}:\langle x, f\rangle=\|x\| \cdot\|f\|,\|f\|=\|x\|\right\}, \quad x \in E .
$$

In the sequel we denote the collection of all fuzzy sets on $E$ by $\mathscr{F}(E)=\{f: E \rightarrow[0,1]\}$. A mapping $A$ from $E$ to $\mathscr{F}(E)$ is called a fuzzy mapping. If $A: E \rightarrow \mathscr{F}(E)$ is a fuzzy mapping, then the set $A(x)$, for $x \in E$, is a fuzzy set in $\mathscr{F}(E)$ (in the sequel we denote $A(x)$ by $\left.A_{x}\right)$ and $A_{x}(y)$, for all $y \in E$ is the degree of membership of $y$ in $A_{x}$.

A fuzzy mapping $A: E \rightarrow \mathscr{F}(E)$ is said to be closed, if for each $x \in E$, the function $y \mapsto$ $A_{x}(y)$ is upper semicontinuous, that is, for any given net $\left\{y_{\alpha}\right\} \subset E$ satisfying $y_{\alpha} \rightarrow y_{0} \in E$, we have $\limsup \sup _{\alpha} A_{x}\left(y_{\alpha}\right) \leq A_{x}\left(y_{0}\right)$. For $f \in \mathscr{F}(E)$ and $\lambda \in[0,1]$, the set

$$
(f)_{\lambda}=\{x \in E: f(x) \geq \lambda\}
$$

is called a $\lambda$-cut set of $f$.

A closed fuzzy mapping $A: E \rightarrow \mathscr{F}(E)$ is said to satisfy condition $(*)$, if there exists a function $a: E \rightarrow[0,1]$ such that for each $x \in E$ the set

$$
\left(A_{x}\right)_{a(x)}=\left\{y \in E: A_{x}(y) \geq a(x)\right\}
$$

is a nonempty bounded subset of $E$. It is clear that if $A$ is a closed fuzzy mapping satisfying condition $\left(^{*}\right)$, then for each $x \in E$, the set $\left(A_{x}\right)_{a(x)} \in \mathrm{CB}(E)$. In fact, let $\left\{y_{\alpha}\right\}_{\alpha \in \Gamma} \subset\left(A_{x}\right)_{a(x)}$ 
be a net and $y_{\alpha} \rightarrow y_{0} \in E$, then $\left(A_{x}\right)\left(y_{\alpha}\right) \geq a(x)$ for each $\alpha \in \Gamma$. Since $A$ is closed, we have

$$
A_{x}\left(y_{0}\right) \geq \limsup _{\alpha \in \Gamma} A_{x}\left(y_{\alpha}\right) \geq a(x) .
$$

This implies that $y_{0} \in\left(A_{x}\right)_{a(x)}$ and so $\left(A_{x}\right)_{a(x)} \in \mathrm{CB}(E)$.

Definition 2.1. Let $T: D(T) \subset E \rightarrow 2^{E}$ be a set-valued mapping.

(1) The mapping $T$ is said to be accretive if for any $x, y \in D(T), u \in T x, v \in T y$, there exists an $j(x-y) \in J(x-y)$ such that

$$
\langle u-v, j(x-y)\rangle \geq 0 \text {. }
$$

(2) The mapping $T$ is said to be $m$-accretive, if $T$ is accretive and $(I+\varrho T)(D(T))=E$ for every (equivalently, for some) $\varrho>0$, where $I$ is the identity mapping.

Remark 2.2. It is well known that if $E=E^{*}=H$ is a Hilbert space, then the notion of accretive mapping coincides with the notion of monotone mapping [7].

Thus we have the following.

Proposition 2.3 (Barbu [7, page 74]). If $E=H$ is a Hilbert space, then $T: D(T) \subset H \rightarrow$ $2^{H}$ is an m-accretive mapping if and only if $T: D(T) \subset H \rightarrow 2^{H}$ is a maximal monotone mapping.

Problem 2.4. Let $E$ be a real Banach space. Let $T, V, Z: E \rightarrow \mathscr{F}(E)$ be three closed fuzzy mappings satisfying condition (*) with functions $a, b, c: E \rightarrow[0,1]$, respectively, and let $g: E \rightarrow E$ be a single-valued and surjective mapping. Let $A: E \times E \rightarrow 2^{E}$ be an $m$-accretive mapping with respect to the first argument. For a given nonlinear mapping $N(\cdot, \cdot): E \times$ $E \rightarrow E$, we consider the problem of finding $u, w, y, z \in E$ such that

$$
\begin{gathered}
T_{u}(w) \geq a(u), \quad V_{u}(y) \geq b(u), \quad Z_{u}(z) \geq c(u), \\
\text { that is, } w \in\left(T_{u}\right)_{a(u)}, \quad y \in\left(V_{u}\right)_{b(u)}, \quad z \in\left(Z_{u}\right)_{c(u)}, \\
\theta \in N(w, y)+A(g(u), z) .
\end{gathered}
$$

The problem (2.7) is called the fuzzy multivalued variational inclusion in Banach spaces.

Now we consider some special cases of problem (2.7).

(1) If $A(g(u), v)=A(g(u)), \forall v \in E$, then the problem (2.7) is equivalent to finding $u, w, y \in E$ such that

$$
\begin{gathered}
T_{u}(w) \geq a(u), \quad V_{u}(y) \geq b(u), \\
\theta \in N(w, y)+A(g(u)) .
\end{gathered}
$$

In the case of classical multivalued mappings, problem (2.8) has been considered and studied by Chang et al. $[10,11,13,15]$. 
(2) If $E=H$ is a Hilbert space, $A: H \times H \rightarrow H$ is a maximal monotone mapping with respect to the first argument and $Z: E \rightarrow \mathscr{F}(E)$ is a closed fuzzy mapping satisfying condition $\left(^{*}\right)$ with $c(x)=1, \forall x \in E$, and it also satisfies the following condition:

$$
Z_{x}=\chi_{\{x\}}, \quad \forall x \in E,
$$

where $\chi_{\{x\}}$ is the characteristic function of the set $\{x\}$, then by Proposition 2.7, $A$ is an $m$-accretive mapping with respect to the first argument. Thus problem (2.7) is equivalent to finding $u, w, y \in H$, such that

$$
T_{u}(w) \geq a(u), \quad V_{u}(y) \geq b(u), \quad \theta \in N(w, y)+A(g(u), u) .
$$

This problem is called the fuzzy multivalued quasi-variational inclusion. In the case of classical multivalued mapping this was introduced and studied in [37, 39-41] by using the resolvent equation technique.

(3) If $E=H$ is a Hilbert space and for any given $x \in H, A(\cdot, x)=\partial \varphi(\cdot, x): H \rightarrow 2^{H}$ is the subdifferential of a proper, convex and lower semicontinuous functional $\varphi(\cdot, x): H \rightarrow$ $R \cup\{+\infty\}$ with respect to the first argument, then problem (2.10) is equivalent to finding $u, w, y \in H$ such that

$$
\begin{gathered}
T_{u}(w) \geq a(u), \quad V_{u}(y) \geq b(u), \\
\langle N(w, y), g(v)-g(u)\rangle+\varphi(g(v), u)-\varphi(g(u), u) \geq 0, \quad \forall v \in H,
\end{gathered}
$$

which is called the multivalued mixed quasi-variational inequality for fuzzy mapping. Some special cases have been considered in $[33,35,38]$.

(4) If the function $\varphi(\cdot, \cdot)$ is the indicator function of a closed convex-valued set $K(u)$ in $H$, that is,

$$
\varphi(u, u)=I_{K(u)}(u)= \begin{cases}0 & \text { if } u \in K(u) \\ +\infty & \text { otherwise }\end{cases}
$$

then problem (2.10) is equivalent to finding $u, w, y \in H$ such that

$$
\begin{gathered}
T_{u}(w) \geq a(u), \quad V_{u}(y) \geq b(u), \\
\langle N(w, y), g(v)-g(u)\rangle \geq 0, \quad \forall v \in K(u) .
\end{gathered}
$$

This problem is called the multivalued quasi-variational inequality for fuzzy mappings. In the case of classical multivalued mappings this problem has been considered by Noor $[37,39]$, using the projection method and the implicit Wiener-Hopf equation technique.

(5) If $K^{*}(u)=\{x \in H,\langle x, v\rangle \geq 0, \forall v \in K(u)\}$ is a polar cone of the convex-valued cone $K(u)$ in $H$, then problem (2.13) is equivalent to finding $u, w, y \in H$ such that

$$
\begin{gathered}
T_{u}(w) \geq a(u), \quad V_{u}(y) \geq b(u), \\
g(u) \in K(u), \quad N(w, y) \in K^{*}(u), \quad\langle N(w, y), g(u)\rangle=0 .
\end{gathered}
$$

This problem is called the multivalued implicit complementarity problem for fuzzy map- 
ping (see, Chang [8] and Chang, Huang [14]). In the case of classical multivalued mappings we refer the reader to $[37,39]$.

As a result we see that for a suitable choice of the fuzzy mappings $T, V, Z$, mappings $A$, $g, N$, and space $E$, we can obtain a number of known and new classes of (fuzzy) variational inequalities, (fuzzy) variational inclusions, and the corresponding (fuzzy) optimization problems from the fuzzy multivalued variational inclusion (2.7).

Related to the fuzzy multivalued variational inclusion (2.7), we now consider its corresponding fuzzy resolvent operator equations. For this purpose we recall some definitions and notions.

Definition 2.5 [7]. Let $A: D(A) \subset E \rightarrow 2^{E}$ be an $m$-accretive mapping. For any given $\rho>0$, the mapping $J_{A}: E \rightarrow D(A)$ associated with $A$ defined by

$$
J_{A}(u)=(I+\varrho A)^{-1}(u), \quad u \in E,
$$

is called the resolvent operator of $A$.

Remark 2.6. Barbu [7, page 72] pointed out that if $A$ is an $m$-accretive mapping, then for every $\rho>0$ the operator $(I+\rho A)^{-1}$ is well defined, single-valued and nonexpansive on the range $R(I+\rho A)$, that is,

$$
\left\|J_{A}(x)-J_{A}(y)\right\| \leq\|x-y\|, \quad \forall x, y \in R(I+\rho A) .
$$

From Remark 2.6 we have the following result.

Proposition 2.7. Let $A(\cdot, \cdot): E \times E \rightarrow 2^{E}$ be an m-accretive mapping with respect to the first argument. For a constant $\rho>0$, let

$$
J_{A(\cdot, z)}=(I+\rho A(\cdot, z))^{-1}, \quad z \in E .
$$

Then for any given $z \in E$, the resolvent operator $J_{A(\cdot, z)}$ is well defined, single-valued, and nonexpansive, that is,

$$
\left\|J_{A(\cdot, z)}(x)-J_{A(\cdot, z)}(y)\right\| \leq\|x-y\|, \quad \forall x, y \in E .
$$

Definition 2.8. Let $T, V: E \rightarrow \mathscr{F}(E)$ be two closed fuzzy mappings satisfying condition $\left(^{*}\right)$ with functions $a, b: E \rightarrow[0,1]$, respectively, and let $N(\cdot, \cdot): E \times E \rightarrow E$ be a nonlinear mapping.

(1) The mapping $x \mapsto N(x, y)$ is said to be $\beta$-Lipschitzian continuous with respect to the fuzzy mapping $T$ if for any $x_{1}, x_{2} \in E$ and $w_{1} \in\left(T_{x_{1}}\right)_{a\left(x_{1}\right)}, w_{2} \in\left(T_{x_{2}}\right)_{a\left(x_{2}\right)}$,

$$
\left\|N\left(w_{1}, y\right)-N\left(w_{2}, y\right)\right\| \leq \beta\left\|x_{1}-x_{2}\right\|, \quad y \in E,
$$

where $\beta>0$ is a constant.

(2) The mapping $y \mapsto N(x, y)$ is said to be $\gamma$-Lipschitzian continuous with respect to the fuzzy mapping $V$ if for any $u_{1}, u_{2} \in E$ and $v_{1} \in\left(V_{u_{1}}\right)_{b\left(u_{1}\right)}, v_{2} \in\left(V_{u_{2}}\right)_{b\left(u_{2}\right)}$,

$$
\left\|N\left(x, v_{1}\right)-N\left(x, v_{2}\right)\right\| \leq \gamma\left\|u_{1}-u_{2}\right\|, \quad x \in E,
$$

where $\gamma>0$ is a constant. 
Definition 2.9. Let $T: E \rightarrow \mathscr{F}(E)$ be a closed fuzzy mapping satisfying condition $(*)$ with a function $a: H \rightarrow[0,1]$ and let $D(\cdot, \cdot)$ be the Hausdorff metric on $\mathrm{CB}(E) . T$ is said to be $\xi$-Lipschitzian continuous if for any $x, y \in E$,

$$
D\left(\left(T_{x}\right)_{a(x)},\left(T_{y}\right)_{a(y)}\right) \leq \xi\|x-y\|,
$$

where $\xi>0$ is a constant.

Related to the fuzzy multivalued variational inclusion (2.7), we consider the following problem.

Find $x, u, w, y, z \in E$ such that

$$
\begin{gathered}
\left(T_{u}\right)(w) \geq a(u), \quad\left(V_{u}\right)(y) \geq b(u), \quad\left(Z_{u}\right)(z) \geq c(u), \\
N(w, y)+\rho^{-1} F_{A(\cdot, z)}(x)=0,
\end{gathered}
$$

where $\rho>0$ is a constant and $F_{A(\cdot, z)}=\left(I-J_{A(\cdot, z)}\right)$, where $I$ is the identity operator and $J_{A(\cdot, z)}$ is the resolvent operator of $A(\cdot, z)$. An equation of the type (2.22) is called the fuzzy resolvent operator equation in Banach spaces. The following two lemmas play an important role in proving our main results.

Lemma 2.10 [9]. Let $E$ be a real Banach space and let $J: E \rightarrow 2^{E^{*}}$ be the normalized duality mapping. Then, for any $x, y \in E$,

$$
\|x+y\|^{2} \leq\|x\|^{2}+2\langle y, j(x+y)\rangle
$$

for all $j(x+y) \in J(x+y)$.

LEMMA 2.11. The following conclusions are equivalent:

(i) $(u, w, y, z)$, where $u \in E,\left(T_{u}\right)(w) \geq a(u),\left(V_{u}\right)(y) \geq b(u),\left(Z_{u}\right)(z) \geq c(u)$ is a solution of the fuzzy multivalued variational inclusion (2.7);

(ii) $(u, w, y, z)$, where $u \in E,\left(T_{u}\right)(w) \geq a(u),\left(V_{u}\right)(y) \geq b(u),\left(Z_{u}\right)(z) \geq c(u)$ is a solution of the following equation:

$$
g(u)=J_{A(\cdot, z)}(g(u)-\rho N(w, y)) ;
$$

(iii) $(x, u, w, y, z), x, u \in E,\left(T_{u}\right)(w) \geq a(u),\left(V_{u}\right)(y) \geq b(u),\left(Z_{u}\right)(z) \geq c(u)$ is a solution of the fuzzy resolvent operator equation (2.22), where

$$
x=g(u)-\rho N(w, y), \quad g(u)=J_{A(\cdot, z)}(x) .
$$

Proof. (i) $\Rightarrow$ (ii). If $(u, w, y, z)$, where $u \in E,\left(T_{u}\right)(w) \geq a(u),\left(V_{u}\right)(y) \geq b(u),\left(Z_{u}\right)(z) \geq$ $c(u)$ is a solution of the fuzzy multivalued variational inclusion $(2.7)$, then we have

$$
\theta \in N(w, y)+A(g(u), z)
$$

Therefore we have

$$
\theta \in-[g(u)-\rho N(w, y)]+[I+\rho A(\cdot, z)](g(u)),
$$


that is,

$$
g(u)=[I+\rho A(\cdot, z)]^{-1}(g(u)-\rho N(w, y))=J_{A(\cdot, z)}(g(u)-\rho N(w, y)) .
$$

(ii) $\Rightarrow$ (iii). Taking $x=g(u)-\rho N(w, y)$, from (2.24) we have $g(u)=J_{A(\cdot, z)}(x)$, and so we have

$$
x=J_{A(\cdot, z)}(x)-\rho N(w, y)
$$

This implies that

$$
N(w, y)+\rho^{-1}\left(I-J_{A(\cdot, z)}\right)(x)=\theta .
$$

Consequently, $(x, u, w, y, z)$ is a solution of the fuzzy resolvent operator equation (2.22).

(iii) $\Rightarrow$ (i). From (2.25) we have

$$
g(u)=J_{A(\cdot, z)}(g(u)-\rho N(w, y)) .
$$

This implies that

$$
g(u)-\rho N(w, y) \in[I+\rho A(\cdot, z)](g(u)),
$$

that is,

$$
\theta \in N(w, y)+A(g(u), z)
$$

Therefore $(u, w, y, z)$, where $u \in E,\left(T_{u}\right)(w) \geq a(u),\left(V_{u}\right)(y) \geq b(u),\left(Z_{u}\right)(z) \geq c(u)$ is a solution of the fuzzy multivalued variational inclusion (2.7).

This completes the proof.

We now invoke Lemma 2.11 and (2.25) to suggest the following algorithms for solving the fuzzy multivalued variational inclusion (2.7) in Banach spaces.

Algorithm 2.12. For any given $x_{0}, u_{0} \in E, w_{0} \in\left(T_{u_{0}}\right)_{a\left(u_{0}\right)}, y_{0} \in\left(V_{u_{0}}\right)_{b\left(u_{0}\right)}, z_{0} \in\left(Z_{u_{0}}\right)_{c\left(u_{0}\right)}$, let

$$
x_{1}=g\left(u_{0}\right)-\rho N\left(w_{0}, y_{0}\right) \text {. }
$$

Since $g$ is surjective, there exists $u_{1} \in E$ such that

$$
g\left(u_{1}\right)=J_{A\left(\cdot, z_{0}\right)}\left(x_{1}\right) .
$$

Since $w_{0} \in\left(T_{u_{0}}\right)_{a\left(u_{0}\right)}, y_{0} \in\left(V_{u_{0}}\right)_{b\left(u_{0}\right)}, z_{0} \in\left(Z_{u_{0}}\right)_{c\left(u_{0}\right)}$, by Nadler [30, page 480], there exist $w_{1} \in\left(T_{u_{1}}\right)_{a\left(u_{1}\right)}, y_{1} \in\left(V_{u_{1}}\right)_{b\left(u_{1}\right)}, z_{1} \in\left(Z_{u_{1}}\right)_{c\left(u_{1}\right)}$, such that

$$
\begin{aligned}
& \left\|w_{0}-w_{1}\right\| \leq(1+1) D\left(\left(T_{u_{0}}\right)_{a\left(u_{0}\right)},\left(T_{u_{1}}\right)_{a\left(u_{1}\right)}\right), \\
& \left\|y_{0}-y_{1}\right\| \leq(1+1) D\left(\left(V_{u_{0}}\right)_{b\left(u_{0}\right)},\left(V_{u_{1}}\right)_{b\left(u_{1}\right)}\right), \\
& \left\|z_{0}-z_{1}\right\| \leq(1+1) D\left(\left(Z_{u_{0}}\right)_{c\left(u_{0}\right)},\left(Z_{u_{1}}\right)_{c\left(u_{1}\right)}\right),
\end{aligned}
$$


where $D$ is the Hausdorff metric on $\mathrm{CB}(E)$. Let

$$
x_{2}=g\left(u_{1}\right)-\rho N\left(w_{1}, y_{1}\right) .
$$

Again by the surjectivity of $g$, there exists $u_{2} \in E$ such that

$$
g\left(u_{2}\right)=J_{A\left(\cdot, z_{1}\right)}\left(x_{2}\right) .
$$

Again by Nadler [30, page 480], there exist $w_{2} \in\left(T_{u_{2}}\right)_{a\left(u_{2}\right)}, y_{2} \in\left(V_{u_{2}}\right)_{b\left(u_{2}\right)}, z_{2} \in\left(Z_{u_{2}}\right)_{c\left(u_{2}\right)}$, such that

$$
\begin{aligned}
& \left\|w_{1}-w_{2}\right\| \leq\left(1+\frac{1}{2}\right) D\left(\left(T_{u_{1}}\right)_{a\left(u_{1}\right)},\left(T_{u_{2}}\right)_{a\left(u_{2}\right)}\right), \\
& \left\|y_{1}-y_{2}\right\| \leq\left(1+\frac{1}{2}\right) D\left(\left(V_{u_{1}}\right)_{b\left(u_{1}\right)},\left(V_{u_{2}}\right)_{b\left(u_{2}\right)}\right), \\
& \left\|z_{1}-z_{2}\right\| \leq\left(1+\frac{1}{2}\right) D\left(\left(Z_{u_{1}}\right)_{c\left(u_{1}\right)},\left(Z_{u_{2}}\right)_{b\left(u_{2}\right)}\right) .
\end{aligned}
$$

Continuing in this way, we can obtain the sequences $\left\{x_{n}\right\},\left\{u_{n}\right\},\left\{w_{n}\right\},\left\{y_{n}\right\},\left\{z_{n}\right\} \subset E$ such that

(i) $w_{n} \in\left(T_{u_{n}}\right)_{a\left(u_{n}\right)}, \quad\left\|w_{n}-w_{n+1}\right\| \leq\left(1+\frac{1}{n+1}\right) D\left(\left(T_{u_{n}}\right)_{a\left(u_{n}\right)},\left(T_{u_{n+1}}\right)_{a\left(u_{n+1}\right)}\right)$,

(ii) $y_{n} \in\left(V_{u_{n}}\right)_{b\left(u_{n}\right)}, \quad\left\|y_{n}-y_{n+1}\right\| \leq\left(1+\frac{1}{n+1}\right) D\left(\left(V_{u_{n}}\right)_{b\left(u_{n}\right)},\left(V_{u_{n+1}}\right)_{b\left(u_{n+1}\right)}\right)$,

(iii) $z_{n} \in\left(Z_{u_{n}}\right)_{c\left(u_{n}\right)}, \quad\left\|z_{n}-z_{n+1}\right\| \leq\left(1+\frac{1}{n+1}\right) D\left(\left(Z_{u_{n}}\right)_{c\left(u_{n}\right)},\left(Z_{u_{n+1}}\right)_{c\left(u_{n+1}\right)}\right)$,

(iv) $x_{n+1}=g\left(u_{n}\right)-\rho N\left(w_{n}, y_{n}\right)$,

(v) $g\left(u_{n+1}\right)=J_{A\left(\cdot, z_{n}\right)}\left(x_{n+1}\right)$,

for all $n \geq 0$.

If $E=H$ is a Hilbert space and $A(\cdot, z)=\partial \varphi(\cdot, z)$, where $\varphi(\cdot, z)$ is the indicator function of a closed convex subset $K$ of $H$, then $J_{A(\cdot, z)}=P_{K}(z)$ (the projection of $H$ onto $K$ ). Then Algorithm 2.12 is reduced to the following.

Algorithm 2.13. For any given $x_{0}, u_{0} \in H, w_{0} \in\left(T_{u_{0}}\right)_{a\left(u_{0}\right)}, y_{0} \in\left(V_{u_{0}}\right)_{b\left(u_{0}\right)}, z_{0} \in\left(Z_{u_{0}}\right)_{c\left(u_{0}\right)}$, compute the sequences $\left\{x_{n}\right\},\left\{u_{n}\right\},\left\{w_{n}\right\},\left\{y_{n}\right\},\left\{z_{n}\right\} \subset H$ by the iterative schemes such that

(i) $w_{n} \in\left(T_{u_{n}}\right)_{a\left(u_{n}\right)}, \quad\left\|w_{n}-w_{n+1}\right\| \leq\left(1+\frac{1}{n+1}\right) D\left(\left(T_{u_{n}}\right)_{a\left(u_{n}\right)},\left(T_{u_{n+1}}\right)_{a\left(u_{n+1}\right)}\right)$,

(ii) $y_{n} \in\left(V_{u_{n}}\right)_{b\left(u_{n}\right)}, \quad\left\|y_{n}-y_{n+1}\right\| \leq\left(1+\frac{1}{n+1}\right) D\left(\left(V_{u_{n}}\right)_{b\left(u_{n}\right)},\left(V_{u_{n+1}}\right)_{b\left(u_{n+1}\right)}\right)$,

(iii) $z_{n} \in\left(Z_{u_{n}}\right)_{c\left(u_{n}\right)}, \quad\left\|z_{n}-z_{n+1}\right\| \leq\left(1+\frac{1}{n+1}\right) D\left(\left(Z_{u_{n}}\right)_{c\left(u_{n}\right)},\left(Z_{u_{n+1}}\right)_{c\left(u_{n+1}\right)}\right)$, 
(iv) $x_{n+1}=g\left(u_{n}\right)-\rho N\left(w_{n}, y_{n}\right)$,

(v) $g\left(u_{n+1}\right)=P_{K}\left(x_{n+1}\right)$.

\section{Main results}

Theorem 3.1. Let $E$ be a real Banach space, let $T, V, Z: E \rightarrow \mathscr{F}(E)$ be three closed fuzzy mappings satisfying condition (*) with functions $a, b, c: E \rightarrow[0,1]$, respectively, let $N(\cdot, \cdot)$ : $E \times E \rightarrow E$ be a single-valued continuous mapping, let $g: E \rightarrow E$ be a single-valued and surjective mapping, and let $A(\cdot, \cdot): E \rightarrow 2^{E}$ be an m-accretive mapping with respect to the first argument satisfying the following conditions:

(i) $g$ is $\delta$-Lipschitzian continuous and $k$-strongly accretive, $0<k<1$;

(ii) $T, V, Z: E \rightarrow \mathscr{F}(E)$ are Lipschitzian continuous fuzzy mappings with Lipschitzian constants $\mu, \xi, \eta$, respectively;

(iii) the mapping $x \mapsto N(x, y)$ is $\beta$-Lipschitzian continuous with respect to the fuzzy mapping $T$ for any given $y \in E$;

(iv) the mapping $y \mapsto N(x, y)$ is $\gamma$-Lipschitzian continuous with respect to the fuzzy mapping $V$ for any given $x \in E$;

here $\delta, \mu, \xi, \beta$, $\eta$, and $\gamma$ all are positive constants.

If the following conditions are satisfied

(a) $\left\|J_{A(\cdot, x)}(z)-J_{A(\cdot, y)}(z)\right\| \leq \sigma\|x-y\| \quad \forall x, y, z \in E, \sigma>0$,

(b)

$$
\begin{aligned}
& 0<\rho<\sqrt{\frac{3+2 k-4 \delta^{2}-2 \sigma^{2} \eta^{2}}{8\left(\gamma^{2}+\beta^{2}\right)}}, \\
& 0<\frac{4 \delta^{2}+2 \sigma^{2} \eta^{2}+8 \rho^{2}\left(\gamma^{2}+\beta^{2}\right)-3}{2}<k<1,
\end{aligned}
$$

then there exist $x, u \in E, w \in\left(T_{u}\right)_{a(u)}, y \in\left(V_{u}\right)_{b(u)}, z \in\left(Z_{u}\right)_{c(u)}$ satisfying the operator equation (2.24), and so $(u, w, y, z)$ is a solution of the fuzzy multivalued variational inclusion (2.7) and the iterative sequences $\left\{x_{n}\right\},\left\{u_{n}\right\},\left\{w_{n}\right\},\left\{y_{n}\right\}$, and $\left\{z_{n}\right\}$ generated by Algorithm 2.12 converge strongly to $x, u, w, y, z$ in $E$, respectively.

Proof. Condition (i) and Lemma 2.10 imply, for any $j\left(u_{n+1}-u_{n}\right) \in J\left(u_{n+1}-u_{n}\right)$, that we have

$$
\begin{aligned}
\| u_{n+1} & -u_{n} \|^{2} \\
& =\left\|g\left(u_{n+1}\right)-g\left(u_{n}\right)-g\left(u_{n+1}\right)+g\left(u_{n}\right)-u_{n+1}+u_{n}\right\|^{2} \\
& \leq\left\|g\left(u_{n+1}\right)-g\left(u_{n}\right)\right\|^{2}-2\left\langle g\left(u_{n+1}\right)-g\left(u_{n}\right)+u_{n+1}-u_{n}, j\left(u_{n+1}-u_{n}\right)\right\rangle \\
& \leq\left\|g\left(u_{n+1}\right)-g\left(u_{n}\right)\right\|^{2}-2(1+k)\left\|u_{n+1}-u_{n}\right\|^{2},
\end{aligned}
$$

so

$$
\left\|u_{n+1}-u_{n}\right\|^{2} \leq \frac{1}{3+2 k}\left\|g\left(u_{n+1}\right)-g\left(u_{n}\right)\right\|^{2}
$$


From (iv) and (v) in (2.40), we have

$$
\begin{aligned}
& \left\|g\left(u_{n+1}\right)-g\left(u_{n}\right)\right\|^{2} \\
& \quad=\left\|J_{A\left(\cdot, z_{n}\right)}\left(g\left(u_{n}\right)-\rho N\left(w_{n}, y_{n}\right)\right)-J_{A\left(\cdot, z_{n-1}\right)}\left(g\left(u_{n-1}\right)-\rho N\left(w_{n-1}, y_{n-1}\right)\right)\right\|^{2} .
\end{aligned}
$$

Now since

$$
\|x+y\|^{2} \leq 2\left(\|x\|^{2}+\|y\|^{2}\right), \quad \forall x, y \in E
$$

we have from condition (a), condition (iii) of (2.40) and condition (i) that

$$
\begin{aligned}
& \frac{1}{2}\left\|g\left(u_{n+1}\right)-g\left(u_{n}\right)\right\|^{2} \\
& \leq\left\|J_{A\left(\cdot, z_{n}\right)}\left(g\left(u_{n}\right)-\rho N\left(w_{n}, y_{n}\right)\right)-J_{A\left(\cdot, z_{n}\right)}\left(g\left(u_{n-1}\right)-\rho N\left(w_{n-1}, y_{n-1}\right)\right)\right\|^{2} \\
&+\left\|J_{A\left(\cdot, z_{n}\right)}\left(g\left(u_{n-1}\right)-\rho N\left(w_{n-1}, y_{n-1}\right)\right)-J_{A\left(\cdot, z_{n-1}\right)}\left(g\left(u_{n-1}\right)-\rho N\left(w_{n-1}, y_{n-1}\right)\right)\right\|^{2} \\
& \leq\left\|g\left(u_{n}\right)-g\left(u_{n-1}\right)-\rho N\left(w_{n}, y_{n}\right)-N\left(w_{n-1}, y_{n-1}\right)\right\|^{2}+\sigma^{2}\left\|z_{n}-z_{n-1}\right\|^{2} \\
& \leq 2 \delta^{2}\left\|u_{n}-u_{n-1}\right\|^{2}+2 \rho^{2}\left\|N\left(w_{n}, y_{n}\right)-N\left(w_{n-1}, y_{n-1}\right)\right\|^{2} \\
&+\sigma^{2}\left(1+\frac{1}{n}\right)^{2} D^{2}\left(\left(Z_{u_{n-1}}\right)_{c\left(u_{n-1}\right)},\left(Z_{u_{n}}\right)_{c\left(u_{n}\right)}\right) .
\end{aligned}
$$

Now we consider the second term on the right-hand side of (3.6). By conditions (iii) and (iv) we have

$$
\begin{aligned}
2 \rho^{2} \| & N\left(w_{n}, y_{n}\right)-N\left(w_{n-1}, y_{n-1}\right) \|^{2} \\
& =2 \rho^{2}\left\|N\left(w_{n}, y_{n}\right)-N\left(w_{n}, y_{n-1}\right)+N\left(w_{n}, y_{n-1}\right)-N\left(w_{n-1}, y_{n-1}\right)\right\|^{2} \\
& \leq 4 \rho^{2}\left\{\left\|N\left(w_{n}, y_{n}\right)-N\left(w_{n}, y_{n-1}\right)\right\|^{2}+\left\|N\left(w_{n}, y_{n-1}\right)-N\left(w_{n-1}, y_{n-1}\right)\right\|^{2}\right\} \\
& \leq 4 \rho^{2}\left\{\gamma^{2}\left\|u_{n}-u_{n-1}\right\|^{2}+\beta^{2}\left\|u_{n}-u_{n-1}\right\|^{2}\right\}=4 \rho^{2}\left\{\gamma^{2}+\beta^{2}\right\}\left\|u_{n}-u_{n-1}\right\|^{2} .
\end{aligned}
$$

Now we consider the third term on the right-hand side of (3.6). By condition (ii) we have

$$
\sigma^{2}\left(1+\frac{1}{n}\right)^{2} D^{2}\left(\left(Z_{u_{n-1}}\right)_{c\left(u_{n-1}\right)},\left(Z_{u_{n}}\right)_{c\left(u_{n}\right)}\right) \leq \sigma^{2}\left(1+\frac{1}{n}\right)^{2} \eta^{2}\left\|u_{n-1}-u_{n}\right\|^{2} .
$$

Substituting (3.7) and (3.8) into (3.6) gives

$$
\frac{1}{2}\left\|g\left(u_{n+1}\right)-g\left(u_{n}\right)\right\|^{2} \leq\left\{2 \delta^{2}+4 \rho^{2}\left(\gamma^{2}+\beta^{2}\right)+\sigma^{2}\left(1+\frac{1}{n}\right)^{2} \eta^{2}\right\}\left\|u_{n}-u_{n-1}\right\|^{2},
$$


that is,

$$
\left\|g\left(u_{n+1}\right)-g\left(u_{n}\right)\right\|^{2} \leq\left\{4 \delta^{2}+8 \rho^{2}\left(\gamma^{2}+\beta^{2}\right)+2 \sigma^{2}\left(1+\frac{1}{n}\right)^{2} \eta^{2}\right\}\left\|u_{n}-u_{n-1}\right\|^{2} .
$$

Substituting (3.10) into (3.3) gives

$$
\left\|u_{n+1}-u_{n}\right\|^{2} \leq \frac{4 \delta^{2}+8 \rho^{2}\left(\gamma^{2}+\beta^{2}\right)+2 \sigma^{2}(1+1 / n)^{2} \eta^{2}}{3+2 k}\left\|u_{n}-u_{n-1}\right\|^{2}
$$

Letting

$$
\begin{gathered}
\alpha_{n}=\sqrt{\frac{4 \delta^{2}+8 \rho^{2}\left(\gamma^{2}+\beta^{2}\right)+2 \sigma^{2}(1+1 / n)^{2} \eta^{2}}{3+2 k}}, \\
\alpha=\sqrt{\frac{4 \delta^{2}+8 \rho^{2}\left(\gamma^{2}+\beta^{2}\right)+2 \sigma^{2} \eta^{2}}{3+2 k}},
\end{gathered}
$$

we have

$$
\left\|u_{n+1}-u_{n}\right\| \leq \alpha_{n}\left\|u_{n}-u_{n-1}\right\|
$$

Obviously, $\alpha_{n} \rightarrow \alpha(n \rightarrow \infty)$. It is easy to prove that condition (b) implies that $0<\alpha<1$, and so $0<\alpha_{n}<1$, when $n$ is sufficiently large. It follows from (3.13) that $\left\{u_{n}\right\}$ is a Cauchy sequence. Let $u_{n} \rightarrow u$. From condition (ii), $T, V, Z: E \rightarrow F(E)$ are $\mu, \xi, \eta$-Lipschitzian continuous fuzzy mappings, respectively, so it follows from (i), (ii), (iii) in (2.40) that $\left\{w_{n}\right\}$, $\left\{y_{n}\right\},\left\{z_{n}\right\}$ are also Cauchy sequences. We can assume that $w_{n} \rightarrow w(n \rightarrow \infty), y_{n} \rightarrow y(n \rightarrow$ $\infty), z_{n} \rightarrow z(n \rightarrow \infty)$. By (iv) and (v) in (2.40) we have

$$
g\left(u_{n+1}\right)=J_{A\left(\cdot, z_{n}\right)}\left[g\left(u_{n}\right)-\rho N\left(w_{n}, y_{n}\right)\right] .
$$

Noting the continuity of $g, N$, and condition (a), let $n \rightarrow \infty$ in the above expression obtain

$$
g(u)=J_{A(\cdot, z)}[g(u)-\rho N(w, y)] .
$$

Finally we prove that $w \in\left(T_{u}\right)_{a(u)}, y \in\left(V_{u}\right)_{b(u)}, z \in\left(Z_{u}\right)_{c(u)}$. Since $w_{n} \in\left(T_{u_{n}}\right)_{a\left(u_{n}\right)}$, we have

$$
\begin{aligned}
\operatorname{dist}\left(w,\left(T_{u}\right)_{a(u)}\right) & \leq\left\|w-w_{n}\right\|+\operatorname{dist}\left(w_{n},\left(T_{u}\right)_{a(u)}\right) \\
& \leq\left\|w-w_{n}\right\|+\operatorname{dist}\left(w_{n},\left(T_{u_{n}}\right)_{a\left(u_{n}\right)}\right)+D\left(\left(T_{u_{n}}\right)_{a\left(u_{n}\right)},\left(T_{u}\right)_{a(u)}\right) \\
& \leq\left\|w-w_{n}\right\|+0+\mu\left\|u_{n}-u\right\| \longrightarrow 0 \quad(n \longrightarrow \infty) .
\end{aligned}
$$

Hence $\operatorname{dist}\left(w,\left(T_{u}\right)_{a(u)}\right)=0$, and so $w \in\left(T_{u}\right)_{a(u)}$, since $\left(T_{u}\right)_{a(u)} \in \mathrm{CB}(E)$.

In a similar way, we can also prove that $y \in\left(V_{u}\right)_{b(u)}$ and $z \in\left(Z_{u}\right)_{c(u)}$. This implies that $(u, w, y, z)$ is a solution of (2.24). By lemma $2.11,(u, w, y, z)$ is a solution of the fuzzy 
multivalued variational inclusion (2.7). Also the iterative sequences $\left\{u_{n}\right\},\left\{w_{n}\right\},\left\{y_{n}\right\}$, $\left\{z_{n}\right\}$ generated by Algorithm 2.12 converge strongly to $u, w, y, z$ in $E$, respectively.

This completes the proof of Theorem 3.1.

Remark 3.2. Theorem 3.1 is a new existence theorem for fuzzy multivalued variational inclusions. The main results by Ding [18, 19], Noor [33, 35, 38], Park and Jeong [43, 44] are special cases of Theorem 3.1. In addition in the case of classical multivalued mappings our results extend and improve the corresponding results in $[32,34,36,37,39-41,47,49$, 45]. Theorem 3.1 also improves and extends the corresponding results in $[10,13,15]$.

The following result can be obtained from Theorem 3.1 immediately.

Theorem 3.3. Let $H$ be a real Hilbert space, let $T, V, Z: E \rightarrow \mathscr{F}(H)$ be three closed fuzzy mappings satisfying condition (*) with functions $a, b, c: E \rightarrow[0,1]$, respectively. Suppose the following conditions are satisfied:

(i) $g: H \rightarrow H$ is a $\delta$-Lipschitzian continuous, surjective, and $k$-strongly monotone mapping, where $k \in(0,1)$ is a constant;

(ii) for any fixed $z \in H, A(\cdot, z)=\partial \varphi(\cdot, z): H \rightarrow 2^{H}$ is a maximal monotone operator with respect to the first argument, where $\varphi(\cdot, \cdot): H \times H \rightarrow \mathbb{R} \cup\{+\infty\}$ is a proper convex lower semicontinuous functional with respect to the first argument;

(iii) $T, V, Z: H \rightarrow \mathscr{F}(H)$ are three Lipschitzian continuous fuzzy mappings with Lipschitzian constants $\mu, \xi, \eta$, respectively;

(iv) $N(\cdot, \cdot): H \times H \rightarrow H$ is a continuous mapping and the mapping $x \mapsto N(x, y)$ is $\beta$ Lipschitzian continuous with respect to the fuzzy mapping $T$;

(v) the mapping $y \mapsto N(x, y)$ is $\gamma$-Lipschitzian continuous with respect to the fuzzy mapping $V$, where $\delta, \mu, \xi, \beta, \gamma$ are all positive constants.

If the following conditions are satisfied:

(a) $\left\|J_{A(\cdot, x)}(z)-J_{A(\cdot, y)}(z)\right\| \leq \sigma\|x-y\| \quad \forall x, y, z \in H, \sigma>0$,

$$
\begin{aligned}
& 0<\rho<\sqrt{\frac{3+2 k-4 \delta^{2}-2 \sigma^{2} \eta^{2}}{8\left(\gamma^{2}+\beta^{2}\right)}}, \\
& 0<\frac{4 \delta^{2}+2 \sigma^{2} \eta^{2}+8 \rho^{2}\left(\gamma^{2}+\beta^{2}\right)-3}{2}<k<1,
\end{aligned}
$$

then there exist $x, u, w, y, z \in H, w \in\left(T_{u}\right)_{a(u)}, y \in\left(V_{u}\right)_{b(u)}, z \in\left(Z_{u}\right)_{c(u)}$ satisfying (2.24) and the iterative sequences $\left\{x_{n}\right\},\left\{u_{n}\right\},\left\{w_{n}\right\},\left\{y_{n}\right\}$, and $\left\{z_{n}\right\}$ generated by Algorithm 2.13 converge strongly to $x, u, w, y, z$ in $H$, respectively.

Remark 3.4. Theorem 3.3 is an improvement and a generalization of the corresponding results by Noor $[33,35,38]$ and Park and Jeong $[43,44]$. Theorem 3.3 is also a fuzzy generalization of the corresponding results by Noor [34, 36, 37, 39-41].

\section{References}

[1] S. Abbasbandy, T. A. Viranloo, Ó. López-Pouso, and J. J. Nieto, Numerical methods for fuzzy differential inclusions, Computers \& Mathematics with Applications 48 (2004), no. 10-11, 16331641. 
[2] R. P. Agarwal, M. F. Khan, D. O’Regan, and Salahuddin, On generalized multivalued nonlinear variational-like inclusions with fuzzy mappings, Advances in Nonlinear Variational Inequalities 8 (2005), no. 1, 41-55.

[3] R. P. Agarwal, D. O'Regan, and V. Lakshmikantham, Viability theory and fuzzy differential equations, Fuzzy Sets and Systems 151 (2005), no. 3, 563-580.

[4] E. E. Ammar and M. L. Hussein, Radiotherapy problem under fuzzy theoretic approach, Chaos, Solitons and Fractals 18 (2003), no. 4, 739-744.

[5] J.-P. Aubin, Mathematical Methods of Game and Economic Theory, Studies in Mathematics and Its Applications, vol. 7, North-Holland, Amsterdam, 1979.

[6] _ Fuzzy differential inclusions, Problems of Control and Information Theory 19 (1990), no. $1,55-67$.

[7] V. Barbu, Nonlinear Semigroups and Differential Equations in Banach Spaces, Noordhoff, Leyden, 1979.

[8] S. S. Chang, Variational Inequality and Complementarity Problems Theory and Applications, Shanghai Scientific and Technological Literature Publishing House, Shanghai, 1991.

[9] Some problems and results in the study of nonlinear analysis, Nonlinear Analysis. Theory, Methods \& Applications 30 (1997), no. 7, 4197-4208.

[10] Set-valued variational inclusions in Banach spaces, Journal of Mathematical Analysis and Applications 248 (2000), no. 2, 438-454.

[11] Existence and approximation of solutions for set-valued variational inclusions in Banach space, Nonlinear Analysis. Theory, Methods \& Applications 47 (2001), no. 1, 583-594.

[12] _ Fuzzy quasivariational inclusions in Banach spaces, Applied Mathematics and Computation 145 (2003), no. 2-3, 805-819.

[13] S. S. Chang, Y. J. Cho, B. S. Lee, and I. H. Jung, Generalized set-valued variational inclusions in Banach spaces, Journal of Mathematical Analysis and Applications 246 (2000), no. 2, 409-422.

[14] S. S. Chang and N.-J. Huang, Generalized complementarity problems for fuzzy mappings, Fuzzy Sets and Systems 55 (1993), no. 2, 227-234.

[15] S. S. Chang, J. K. Kim, and K. H. Kim, On the existence and iterative approximation problems of solutions for set-valued variational inclusions in Banach spaces, Journal of Mathematical Analysis and Applications 268 (2002), no. 1, 89-108.

[16] S. S. Chang and Y. G. Zhu, On variational inequalities for fuzzy mappings, Fuzzy Sets and Systems 32 (1989), no. 3, 359-367.

[17] C. E. Chidume, H. Zegeye, and K. R. Kazmi, Existence and convergence theorems for a class of multi-valued variational inclusions in Banach spaces, Nonlinear Analysis 59 (2004), no. 5, 649656.

[18] X. P. Ding, Generalized implicit quasivariational inclusions with fuzzy set-valued mappings, Computers \& Mathematics with Applications 38 (1999), no. 1, 71-79.

[19] X. P. Ding and J. Y. Park, A new class of generalized nonlinear implicit quasivariational inclusions with fuzzy mappings, Journal of Computational and Applied Mathematics 138 (2002), no. 2, $243-257$.

[20] D. Dubois and H. Prade, Fuzzy Sets and Systems. Theory and Applications, Mathematics in Science and Engineering, vol. 144, Academic Press, London, 1980.

[21] G. Feng and G. Chen, Adaptive control of discrete-time chaotic systems: a fuzzy control approach, Chaos, Solitons and Fractals 23 (2005), no. 2, 459-467.

[22] F. Gu, Iteration processes for approximating fixed points of operators of monotone type, Proceedings of the American Mathematical Society 129 (2001), no. 8, 2293-2300.

[23] M. Guo, X. Xue, and R. Li, Impulsive functional differential inclusions and fuzzy population models, Fuzzy Sets and Systems 138 (2003), no. 3, 601-615.

[24] N.-J. Huang and Y. J. Cho, Generalized strongly set-valued nonlinear complementarity problems, International Journal of Mathematics and Mathematical Sciences 22 (1999), no. 3, 597-604. 


\section{Fuzzy multivalued variational inclusions}

[25] E. Hüllermeier, An approach to modelling and simulation of uncertain dynamical systems, International Journal of Uncertainty, Fuzziness and Knowledge-Based Systems 5 (1997), no. 2, 117-137.

[26] O. Kaleva, Fuzzy differential equations, Fuzzy Sets and Systems 24 (1987), no. 3, 301-317.

[27] V. Lakshmikantham and R. N. Mohapatra, Theory of Fuzzy Differential Equations and Inclusions, Series in Mathematical Analysis and Applications, vol. 6, Taylor \& Francis, London, 2003.

[28] V. Lakshmikantham and J. J. Nieto, Differential equations in metric spaces: an introduction and an application to fuzzy differential equations, Dynamics of Continuous, Discrete \& Impulsive Systems. Series A. Mathematical Analysis 10 (2003), no. 6, 991-1000.

[29] K. K. Majumdar, One dimensional fuzzy differential inclusions, Journal of Intelligent \& Fuzzy Systems 13 (2003), no. 1, 1-5.

[30] S. B. Nadler Jr., Multi-valued contraction mappings, Pacific Journal of Mathematics 30 (1969), $475-488$.

[31] S. Nanda and S. Pani, Fuzzy variational inequality and complementarity problem, Journal of Fuzzy Mathematics 12 (2004), no. 1, 231-235.

[32] J. J. Nieto and A. Torres, Midpoints for fuzzy sets and their application in medicine, Artificial Intelligence in Medicine 27 (2003), no. 1, 81-101.

[33] M. A. Noor, Variational inequalities for fuzzy mappings. I, Fuzzy Sets and Systems 55 (1993), no. 3, 309-312.

[34] _ Generalized set-valued variational inclusions and resolvent equations, Journal of Mathematical Analysis and Applications 228 (1998), no. 1, 206-220.

[35] _ Variational inequalities for fuzzy mappings. II, Fuzzy Sets and Systems 97 (1998), no. 1, $101-107$.

[36] _ Some algorithms for general monotone mixed variational inequalities, Mathematical and Computer Modelling 29 (1999), no. 7, 1-9.

[37] _ Set-valued quasi variational inclusions, The Korean Journal of Computational \& Applied Mathematics 7 (2000), no. 1, 101-113.

[38] _ Variational inequalities for fuzzy mappings. III, Fuzzy Sets and Systems 110 (2000), no. 1, $101-108$.

[39] _ Three-step approximation schemes for multivalued quasi variational inclusions, Nonlinear Functional Analysis and Applications 6 (2001), no. 3, 383-394.

[40] - Multivalued quasi variational inclusions and implicit resolvent equations, Nonlinear Analysis. Theory, Methods \& Applications 48 (2002), no. 2, 159-174.

[41] , Two-step approximation schemes for multivalued quasi variational inclusions, Nonlinear Functional Analysis and Applications 7 (2002), no. 1, 1-14.

[42] D. O'Regan, V. Lakshmikantham, and J. J. Nieto, Initial and boundary value problems for fuzzy differential equations, Nonlinear Analysis 54 (2003), no. 3, 405-415.

[43] J. Y. Park and J. U. Jeong, Generalized strongly quasivariational inequalities for fuzzy mappings, Fuzzy Sets and Systems 99 (1998), no. 1, 115-120.

[44] A A perturbed algorithm of variational inclusions for fuzzy mappings, Fuzzy Sets and Systems 115 (2000), no. 3, 419-424.

[45] Salahuddin, An iterative scheme for a generalized quasivariational inequality, Advances in Nonlinear Variational Inequalities 4 (2001), no. 2, 89-98.

[46] A. H. Siddiqi and R. Ahmad, An iterative algorithm for generalized nonlinear variational inclusions with relaxed strongly accretive mappings in Banach spaces, International Journal of Mathematics and Mathematical Sciences 2004 (2004), no. 17-20, 1035-1045.

[47] L. U. Uko, Strongly nonlinear generalized equations, Journal of Mathematical Analysis and Applications 220 (1998), no. 1, 65-76.

[48] L. A. Zadeh, Fuzzy sets, Information and Computation 8 (1965), 338-353. 
[49] L.-C. Zeng, Iterative algorithm for finding approximate solutions to completely generalized strongly nonlinear quasivariational inequalities, Journal of Mathematical Analysis and Applications 201 (1996), no. 1, 180-194.

[50] H. Y. Zhu, Z. Liu, S. H. Shim, and S. M. Kang, Generalized multivalued quasivariational inclusions for fuzzy mappings, Advances in Nonlinear Variational Inequalities 7 (2004), no. 2, 59-70.

[51] H. I. Zimmermann, Fuzzy Set Theory and Its Applications, Kluwer Academic, Boston, 1965.

S. S. Chang: Department of Mathematics, Yibin University, Yibin, Sichuan 644007, China;

Department of Mathematics, Sichuan University, Chengdu, Sichuan 610064, China

E-mail address: sszhang_1@yahoo.com.cn

D. O’Regan: Department of Mathematics, National University of Ireland, Galway, University Road, Galway, Ireland

E-mail address: donal.oregan@nuigalway.ie

J. K. Kim: Department of Mathematics, Kyungnam University, Masan, Kyungnam 631-701, Korea

E-mail address: jongkyuk@kyungnam.ac.kr 